\title{
Public acceptability of non-pharmaceutical interventions to control a pandemic in the United Kingdom: a discrete choice experiment
}

\begin{abstract}
Authors:
Luis Enrique Loria-Rebolledo, Mandy Ryan, Verity Watson, Mesfin G Genie, Ruben Andreas Sakowsky, Daniel Powell, Shantini Paranjothy

Health Economics Research Unit, University of Aberdeen, UK AB25 2ZD, Luis Enrique LoriaRebolledo research fellow, Health Economics Research Unit, University of Aberdeen, UK AB25 2ZD, Mandy Ryan professor, Health Economics Research Unit, University of Aberdeen, UK AB25 2ZD, Verity Watson senior research fellow, Health Economics Research Unit, University of Aberdeen, UK AB25 2ZD, Mesfin G Genie research fellow, Department of Medical Ethics and History of Medicine, University Medical Center Göttingen, Germany, Ruben Andreas Sakowsky research fellow, Health Data Science Research Centre, University of Aberdeen, UK, AB25 2ZD, Daniel Powell research fellow, Health Psychology, University of Aberdeen, UK, AB25 2ZD, Shantini Paranjothy professor
\end{abstract}

Correspondance to:

Dr Luis E. Loría-Rebolledo

luis.loria@abdn.ac.uk

Health Economics Research Unit, University of Aberdeen, UK, AB25 2ZD

NOTE: This preprint reports new research that has not been certified by peer review and should not be used to guide clinical practice. 


\begin{abstract}
Objective

To understand how individuals make trade-offs between features of lockdown interventions to control a pandemic across the four nations of the United Kingdom.
\end{abstract}

\title{
Design
}

Survey that included a Discrete Choice Experiment (DCE). The survey design was informed using policy documents, social media analysis and with input from remote think aloud interviews with members of the public $(n=23)$.

Setting

Nation-wide survey across the four nations of the United Kingdom. Representative sample in terms of age and sex for each of the nations recruited using an online panel between $29^{\text {th }}$ October and $12^{\text {th }}$ December 2020.

\section{Participants}

Individuals who are over 18 years old. A total of 4120 adults completed the survey (1112 in England, 848 in Northern Ireland, 1143 in Scotland and 1098 in Wales).

\section{Primary outcome measure}

Adult's preferences for, and trade-offs between, type of lockdown restrictions, length of lockdown, postponement of routine healthcare, excess deaths, impact on ability to buy things and unemployment.

\section{$\underline{\text { Results }}$}

In all four countries, one out of five respondents were willing to reduce excess deaths at all costs. The majority of adults are willing to accept higher excess deaths if this means lockdowns that are less strict, shorter and do not postpone routine healthcare. On average, respondents in England were willing to accept a higher increase in excess deaths to have less strict lockdown restrictions introduced compared to Scotland, Northern Ireland, and Wales, respectively.

\section{Conclusions}

The majority of the UK population is willing to accept the increase in excess deaths associated with introducing less strict lockdown restrictions. The acceptability of different restriction scenarios varies according to the features of the lockdown and across countries. Authorities can use information about trade-off preferences to inform the introduction of different lockdown restriction levels, and design compensation policies that maximise societal welfare. 
medRxiv preprint doi: https://doi.org/10.1101/2021.10.12.21264883; this version posted October 14, 2021. The copyright holder for this preprint (which was not certified by peer review) is the author/funder, who has granted medRxiv a license to display the preprint in perpetuity. It is made available under a CC-BY-NC-ND 4.0 International license .

\section{Strengths and limitations of this study}

- This study offers empirical evidence that, unlike existing data from opinion polls and citizens' panels, offers a clear understanding of the trade-offs between restrictions and impacts of lockdown on society.

- Estimating preferences for each nation, and quantifying them in terms of a common denominator, allows a comparison that takes into account the heterogeneity of UK nations and can be used to inform the introduction of different levels of lockdown restrictions in each.

- A limitation of our study is that we are not able to estimate the effect of on-going lockdowns in preferences. Furthermore, our results are not necessarily transferable to other nations.

\section{Introduction}

The COVID-19 pandemic has required countries worldwide to introduce nonpharmaceutical interventions to protect the health and wellbeing of their citizens.[1] The majority of European and high-income nations have focused on reducing the $\mathrm{R}$ number to less than one and thereby curtailing the epidemic spread of the virus.[2,3] This strategy requires a number of non-pharmaceutical interventions such as enforced social distancing across all age groups, closing schools and non-essential businesses, and a range of other social restrictions.[4] This has led to local and nationwide lockdowns and other restrictions to control infection rates and excess deaths within geographically defined populations.[5-7]

Lockdowns have wider indirect impacts on health and wellbeing, and lockdown decisions require a careful balancing of the direct impacts on mortality caused by COVID-19 with the indirect wider health, social and economic impacts.[8-11] Further, lockdown compliance will determine its effectiveness. Compliance is more likely if policies are acceptable. Policies are more likely to be acceptable if the public's preferences are understood and the diversity of view is recognised. The World Health Organization criteria for deciding whether to lift lockdown restrictions is defined as "Communities are fully educated, engaged and empowered to adjust to the "new norm" of everyday life.[12] This criterion requires a better understanding of how the public respond to and value the trade-offs faced during and postpandemic. For example, are the public willing to accept a certain number of excess deaths to have restrictions eased?

Prior to the COVID-19 pandemic, there was limited evidence on the understanding of how people think of lockdown policies in the UK.[13] During the pandemic, public attitudes to government responses to the pandemic have been explored using opinion polls and qualitative studies.[14-16] The Scottish Government and Bank of England established citizen's panels. $[17,18]$ These instruments offer insight into the views and concerns of the population. However, they provide no understanding of the trade-offs that individuals are willing to make. For example, the Scottish citizen's panel recommended that the Scottish Government should implement an elimination strategy, and where this is not feasible, 
medRxiv preprint doi: https://doi.org/10.1101/2021.10.12.21264883; this version posted October 14, 2021. The copyright holder for this preprint (which was not certified by peer review) is the author/funder, who has granted medRxiv a license to display the preprint in perpetuity.

It is made available under a CC-BY-NC-ND 4.0 International license .

should aim for maximum suppression of the virus, but not the cost of the restrictions that were acceptable to achieve this. Thus, we use a preference elicitation instrument tailored to quantify preferences, a discrete choice experiment (DCE), to provide new evidence on the acceptable number of excess deaths to the UK public when easing or tightening restrictions.

\section{Methods}

\section{Study Sample}

We conducted a cross-sectional survey among a representative sample of adults aged over 18 from across the four nations of the United Kingdom. The survey was implemented between $29^{\text {th }}$ October and $12^{\text {th }}$ December 2020. Respondents were recruited using an online survey research panel maintained by the company Qualtrics. The survey was piloted in early October 2020 ( $n=50$ per nation). Respondents were screened by the recruiting company using sex and age using quotas to ensure a balance in each nation. The research company excluded respondents that completed the survey in less than half the median time of completion of the pilot stage of the survey (14 minutes).

\section{Discrete Choice Experiment}

Respondents completed a self-complete online survey that asked about the individual's experience during the COVID-19 pandemic, lockdowns that had occurred, any impacts on their healthcare, their spending ability and employment. The survey included a discrete choice experiment (DCE), a choice-based survey that quantifies preferences for attributes (or features) of goods, services or policies. Respondents completed a series of eight choice tasks based on the features of government restrictions. The hypothetical choice tasks focussed on six features of government restrictions that describe different types of lockdown and their likely health and economic consequences.

Features used to describe the type of lockdown were: restriction severity using a colourbased tier system (Figure 1), length in weeks, and postponement of routine healthcare procedures. The health consequences were the number of excess deaths (we also report infection numbers as a complement based on the infection rate).[19] The economic consequences included respondent's household's ability to buy things (personal impact) and the number of job losses (societal impact). See the online Supplemental Table 1 for the features and associated levels. The features and levels were informed by policy documents,[12] impacts of interventions that were implemented in response to COVID19,[4] literature on preferences for lockdown measures from previous pandemics,[20,21] and a social media analysis. A more detailed description of the development stage can be found in the study's published protocol.[22]

[Figure 1 here] 
medRxiv preprint doi: https://doi.org/10.1101/2021.10.12.21264883; this version posted October 14, 2021. The copyright holder for this preprint (which was not certified by peer review) is the author/funder, who has granted medRxiv a license to display the preprint in perpetuity. It is made available under a CC-BY-NC-ND 4.0 International license .

Lockdown features and levels were combined into pairwise choice tasks using a D-efficient design.[23,24] The design results in 24 tasks. Respondents were allocated randomly to one of the three survey versions, each with eight tasks. Respondents were asked to choose between two lockdown descriptions (Figure 2). The order of the eight tasks was randomised for each respondent to minimise ordering effects.[25]

[Figure 2 here]

\section{Patient and Public Involvement}

Adult members of the public were invited, using two targeted social media campaigns, to take part in the study development stage (see online Supplemental Figures 1-5). These engagements were used to create the survey's content and format, and to construct the framing of the Discrete Choice Experiment's features and levels (see online Supplemental Material document). Twenty-three think-aloud interviews were carried out between the months of June and August 2020. The outcome of each interviews was used iteratively, until saturation was achieved, to make edits to the survey to ensure it captured the intended preferences, was understandable, and minimised respondent burden. The study results will be disseminated to the wider public, with the help of the SAG, using layperson summaries and multimedia content through mass media. Furthermore, the study's Stakeholder Advisory Group (SAG), which includes a member of Scotland's Chief Scientist Office's Public Engagement Group, has been involved since its conception and provided insight into the research questions, overall design and dissemination strategy. Because of the study's ethical approvals, it is not possible for us to contact the members of the public who took part in the survey development stage, nor respondents of the main survey, to disseminate results individually.

\section{Statistical Analysis}

The devolved governments of the UK set their own lockdown policies; therefore, statistical analysis was conducted separately for each of the four devolved nations of the UK. The minimal sample size for the DCE given the eight tasks per respondent, a baseline choice probability of $50 \%$ (given there were two options in each choice set), an accuracy level of $90 \%$ and a confidence level of $95 \%$, using Louviere's formula for choice proportions, was 49 respondents.[22] Given that we aimed to estimate preferences using flexible logit models, we aimed for a conservative size of 1000 per nation in the UK.

We first test if any respondents were unwilling to accept an increase in excess deaths for improvements in other features. This was defined as respondents who always chose the description with the lowest number of excess deaths. The response pattern for these respondents is shown in the online Supplemental Table 2. We estimated a logit regression model to understand the characteristics of this group for each nation. Predictors included: sex, age, self-perceived health, number of children in household, household income quintile, whether they were asked to shield during previous lockdowns, had their main job 
medRxiv preprint doi: https://doi.org/10.1101/2021.10.12.21264883; this version posted October 14, 2021. The copyright holder for this preprint (which was not certified by peer review) is the author/funder, who has granted medRxiv a license to display the preprint in perpetuity. It is made available under a CC-BY-NC-ND 4.0 International license .

impacted (furloughed, reduced hours or made redundant), had caring responsibilities and if they had seen their standard of living worsened during the COVID-19 pandemic.

We then analysed the choice tasks using an errors-component logit model, allowing for the panel structure of the data.[26] Parameter estimates represented the effect of each feature on preferences. The ratio of estimates represents the trade-off between two features. Further, trade-offs between different features, when elicited in terms of a common denominator, can be added to estimate the overall trade-off for a particular lockdown scenario. When elicited in terms of excess deaths, these trade-offs indicate the maximum number of lives that need to be saved to introduce a hypothetical lockdown scenario. For example, how many excess deaths would need to be saved when introducing a four-week strict lockdown that cancels all non-COVID-19 healthcare procedures?

The difference in trade-offs between two lockdown scenarios can be interpreted as the maximum number of excess deaths that would be accepted if the more preferred scenario were introduced. To illustrate how these differences can inform policy, we assume that each nation faces a four-week red level (see Figure 1) restriction lockdown that postpones all non-COVID-19 healthcare procedures, and estimate the acceptable number of excess deaths to have this eased to less strict lockdown scenarios. Specifically we compare easing to 12 different lockdowns made up of combinations of amber and yellow restrictions (Figure 1) that vary in length between 8,10 and 12 weeks, and in whether they postpone healthcare services.

Data was weighted to ensure a representative sample in terms of age and sex using iterative proportional fitting.[27] All logit models were estimated using maximum likelihood techniques using the statistical software $\mathrm{R}$ (version 3.6.3). Standard errors and confidence intervals (Cls) were computed using the delta method.

\section{Results}

4120 respondents completed the survey: 1112 in England, 848 in Northern Ireland, 1143 in Scotland, and 1098 in Wales. Table 1 shows the sample descriptive characteristics across nations.

The number of respondents who consistently chose the alternative with the least excess deaths was 225 (20.2\%) in England, 193 (22.8\%) in Northern Ireland, 262 (22.9\%) in Scotland, and 247 (22.5\%) in Wales. Results from the logit model are shown in Table 2. In England, none of the considered variables were associated with respondents always choosing the lowest number of excess deaths. In Northern Ireland, this response pattern was negatively associated with respondents who experienced an impact on employment (adjusted odds ratio [OR] 0.58 [95\% Cl 0.35-0.97], p=0.04). In Scotland, this response pattern was also negatively associated with respondents who experienced an impact on employment (0.62 [0.40-0.95], $p=0.03$ ), and household income of $£ 20,800-£ 31,200$ compared to the reference level of $£ 0-£ 10,400$ (0.54 [0.31-0.95], $p=0.03$ ). Furthermore, 
this response pattern was positively associated with having a higher education degree (compared to less than higher education) $(1.77$ [1.28-2.45], $p<0.01)$ and fair self-reported health compared to very good (1.82 [1.11-2.97], $\mathrm{p}=0.02)$. In Wales, this response pattern was negatively associated with age over 55 compared to 18-34 (0.63 [0.40-0.98], $p=0.04)$, household incomes of $£ 10,400-£ 20,800$ (0.49 [0.29-0.83], $p=0.01), £ 20,800-£ 31,200$ (0.57 [0.34-0.96], $p=0.04)$ and over $£ 52,000(0.49$ [0.79-1.56], $p=0.03)$ compared to $£ 0-$ $£ 10,400$. Univariate analyses for each factor are shown in the online Supplemental Table 3.

The preference parameter estimates and corresponding trade-offs in terms of excess deaths based on responses to the choice tasks are shown in Table 3. Across the four nations, respondents prefer lockdowns with less strict restrictions (i.e., green and yellow level) to strict ones (i.e., amber and red level), shorter lockdowns, fewer excess deaths, fewer job losses, and less impact on their ability to buy goods. In England, Northern Ireland and Scotland, respondents prefer no postponement of routine healthcare procedures (at the $10 \%$ level). The maximum number of lives (out of 10,000) that need to be saved to accept a change in each of the lockdown features and consequences is shown in the MRS column for each nation.

Figure 3 shows the acceptable maximum excess deaths for easing restrictions from a further 4-week red lockdown to the less strict lockdowns. The highest aversion to strict lockdowns is found in England, followed by Scotland, Northern Ireland and Wales, as seen by the higher number of acceptable excess deaths for lockdown easing. For example, the maximum number of acceptable deaths when easing to an 8-week yellow restriction with no healthcare postponement is $3.62(95 \% \mathrm{Cl} 2.67-4.58)$ in England, $2.22(1.21-3.24)$ in Northern Ireland, 2.41 (1.57-3.24) in Scotland, and 1.10 (0.18-2.02) in Wales. These rates equal $18958,361,1265$, and 323 excess deaths for each nation, respectively.

As expected, the maximum number of acceptable deaths is lower when moving to more strict (e.g., amber over yellow) and longer lockdowns that postpone routine healthcare procedures. For example, the difference in the acceptable number of deaths between a 4week red lockdown and a 12-week amber lockdown with healthcare postponement is 0.85 (0.03-1.67) in England and not statistically different from zero in Northern Ireland $\left(X^{2}=0.88, p=0.35\right)$, Scotland $\left(X^{2}=1.84, p=0.17\right)$, and Wales $\left(X^{2}=0.08, p=0.77\right)$. This suggests that respondents in Northern Ireland, Scotland, and Wales are indifferent between continuing with a further 4-week red restriction and easing to a 12-week amber restriction with healthcare postponement. 
Table 1. Characteristics associated with sample by nation.

\begin{tabular}{|c|c|c|c|c|c|c|c|c|}
\hline \multirow{2}{*}{ Sex } & \multicolumn{2}{|c|}{ England } & \multicolumn{2}{|c|}{$\begin{array}{l}\text { Northern } \\
\text { Ireland }\end{array}$} & \multicolumn{2}{|c|}{ Scotland } & \multicolumn{2}{|c|}{ Wales } \\
\hline & & & & & & & & \\
\hline Female & 556 & $50.0 \%$ & 436 & $51.4 \%$ & 592 & $51.8 \%$ & 562 & $51.2 \%$ \\
\hline Male & 556 & $50.0 \%$ & 412 & $48.6 \%$ & 551 & $48.2 \%$ & 536 & $48.8 \%$ \\
\hline \multicolumn{9}{|l|}{ Age } \\
\hline $18-34$ & 312 & $28.1 \%$ & 242 & $28.5 \%$ & 315 & $27.6 \%$ & 294 & $26.8 \%$ \\
\hline $35-55$ & 373 & $33.5 \%$ & 293 & $34.6 \%$ & 375 & $32.8 \%$ & 343 & $31.2 \%$ \\
\hline $55+$ & 427 & $38.4 \%$ & 313 & $36.9 \%$ & 453 & $39.6 \%$ & 461 & $42.0 \%$ \\
\hline \multicolumn{9}{|l|}{ Health } \\
\hline Very good & 192 & $17.3 \%$ & 140 & $16.5 \%$ & 200 & $17.5 \%$ & 197 & $18.0 \%$ \\
\hline Good & 542 & $48.8 \%$ & 415 & $49.0 \%$ & 567 & $49.6 \%$ & 505 & $46.0 \%$ \\
\hline Fair & 299 & $26.9 \%$ & 217 & $25.6 \%$ & 304 & $26.6 \%$ & 316 & $28.8 \%$ \\
\hline Bad & 59 & $5.3 \%$ & 63 & $7.4 \%$ & 65 & $5.7 \%$ & 65 & $6.0 \%$ \\
\hline Very bad & 20 & $1.8 \%$ & 13 & $1.5 \%$ & 7 & $0.6 \%$ & 15 & $1.3 \%$ \\
\hline \multicolumn{9}{|l|}{ Shield } \\
\hline No & 831 & $74.8 \%$ & 604 & $71.2 \%$ & 947 & $82.9 \%$ & 823 & $74.9 \%$ \\
\hline Yes & 281 & $25.2 \%$ & 244 & $28.8 \%$ & 196 & $17.1 \%$ & 275 & $25.1 \%$ \\
\hline \multicolumn{9}{|l|}{ Adults in household } \\
\hline 1 & 273 & $24.6 \%$ & 192 & $22.6 \%$ & 281 & $24.6 \%$ & 249 & $22.7 \%$ \\
\hline 2 & 614 & $55.3 \%$ & 446 & $52.6 \%$ & 666 & $58.3 \%$ & 657 & $59.8 \%$ \\
\hline 3 & 136 & $12.3 \%$ & 146 & $17.2 \%$ & 136 & $11.9 \%$ & 133 & $12.2 \%$ \\
\hline$>3$ & 88 & $7.9 \%$ & 64 & $7.6 \%$ & 60 & $5.3 \%$ & 58 & $5.3 \%$ \\
\hline \multicolumn{9}{|l|}{ Children in household } \\
\hline 0 & 804 & $72.3 \%$ & 620 & $73.1 \%$ & 861 & $75.3 \%$ & 817 & $74.4 \%$ \\
\hline 1 & 156 & $14.0 \%$ & 119 & $14.0 \%$ & 163 & $14.2 \%$ & 137 & $12.5 \%$ \\
\hline 2 & 116 & $10.4 \%$ & 80 & $9.4 \%$ & 99 & $8.6 \%$ & 107 & $9.8 \%$ \\
\hline$>2$ & 36 & $3.2 \%$ & 29 & $3.4 \%$ & 21 & $1.9 \%$ & 37 & $3.3 \%$ \\
\hline \multicolumn{9}{|l|}{ Household income } \\
\hline$£ 0-£ 10,400$ & 106 & $9.5 \%$ & 112 & $13.2 \%$ & 138 & $12.1 \%$ & 156 & $14.2 \%$ \\
\hline$£ 10,400-£ 20,800$ & 238 & $21.4 \%$ & 185 & $21.8 \%$ & 214 & $18.7 \%$ & 242 & $22.0 \%$ \\
\hline$£ 10,400-£ 31,200$ & 227 & $20.4 \%$ & 204 & $24.0 \%$ & 266 & $23.3 \%$ & 253 & $23.0 \%$ \\
\hline$£ 31,200-£ 52,000$ & 323 & $29.0 \%$ & 221 & $26.1 \%$ & 296 & $25.9 \%$ & 277 & $25.2 \%$ \\
\hline$£ 52,000+$ & 218 & $19.6 \%$ & 125 & $14.8 \%$ & 229 & $20.0 \%$ & 170 & $15.5 \%$ \\
\hline \multicolumn{9}{|l|}{ Education } \\
\hline Less than higher education & 695 & $62.5 \%$ & 504 & $59.4 \%$ & 665 & $58.2 \%$ & 679 & $61.9 \%$ \\
\hline Higher education degree & 417 & $37.5 \%$ & 343 & $40.5 \%$ & 478 & $41.8 \%$ & 419 & $38.2 \%$ \\
\hline \multicolumn{9}{|l|}{ Job impact } \\
\hline No & 820 & $73.7 \%$ & 650 & $76.7 \%$ & 907 & $79.4 \%$ & 830 & $75.6 \%$ \\
\hline Yes & 292 & $26.3 \%$ & 198 & $23.3 \%$ & 236 & $20.6 \%$ & 268 & $24.4 \%$ \\
\hline aring $r$ & & & & & & & & \\
\hline
\end{tabular}


medRxiv preprint doi: https://doi.org/10.1101/2021.10.12.21264883; this version posted October 14, 2021. The copyright holder for this preprint (which was not certified by peer review) is the author/funder, who has granted medRxiv a license to display the preprint in perpetuity. It is made available under a CC-BY-NC-ND 4.0 International license.

$\begin{array}{lcccccccc}\text { No } & 950 & 85.4 \% & 692 & 81.6 \% & 955 & 83.5 \% & 912 & 83.1 \% \\ \quad \text { Yes } & 162 & 14.6 \% & 156 & 18.4 \% & 188 & 16.5 \% & 186 & 16.9 \% \\ \text { Affected usual healthcare } & & & & & & & & \\ \quad \text { No } & 654 & 58.8 \% & 399 & 47.0 \% & 640 & 56.0 \% & 579 & 52.8 \% \\ \quad \text { Yes } & 458 & 41.2 \% & 449 & 53.0 \% & 503 & 44.0 \% & 518 & 47.2 \% \\ \text { Impact on standard of living } & & & & & & & & \\ \quad \begin{array}{l}\text { Worsened } \\ \text { Same or improved }\end{array} & 332 & 29.9 \% & 317 & 37.4 \% & 354 & 31.0 \% & 352 & 32.1 \% \\ & 780 & 70.1 \% & 531 & 62.6 \% & 789 & 69.0 \% & 757 & 69.0 \% \\ \text { Total } & 1112 & & & & & & & \\ \end{array}$

Note: weighted frequencies. 
Table 2. Characteristics associated with respondents that always minimises excess deaths in the discrete choice experiment tasks

\section{England}

\section{Northern Ireland}

Scotland

Wales

Sex

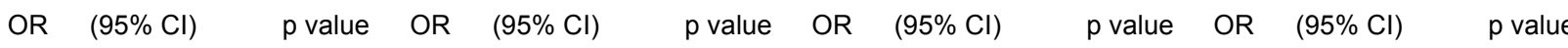

\section{Female}

Male

Age

$18-34$
$35-55$
$55+$

Health

Very good
Good
Fair
Bad
Very bad

Shield

No
Yes

Adults in household

1
2
3
$>3$

$\begin{array}{llllll}1 & \text { (ref) } & & 1 & \text { (ref) } & \\ 0.82 & (0.58-1.16) & 0.27 & 0.81 & (0.55-1.18) & 0.27\end{array}$

$\begin{array}{lll}1 & \text { (ref) } & \\ 1.60 & (1.00-2.53) & 0.05 \\ 1.27 & (0.80-2.02) & 0.32\end{array}$

1 (ref)

$1.54 \quad(0.93-2.56) \quad 0.09$

$1.29 \quad(0.75-2.23) \quad 0.36$

$\begin{array}{lll}1 & \text { (ref) } & \\ 1.26 & (0.77-2.04) & 0.36 \\ 1.52 & (0.90-2.59) & 0.12 \\ 1.36 & (0.59-3.10) & 0.47 \\ 2.16 & (0.67-6.95) & 0.20\end{array}$

1 (ref)

$1.07 \quad(0.62-1.84) \quad 0.81$

$0.92 \quad(0.50-1.71) \quad 0.80$

$1.06 \quad(0.46-2.46) \quad 0.89$

$0.82 \quad(0.19-3.63) \quad 0.80$

$$
1 \text { (ref) }
$$

$1.04(0.75-1.44) \quad 0.83$

1 (ref)

$0.93 \quad(0.61-1.41) \quad 0.73$

$1.33 \quad(0.86-2.06) \quad 0.20$
1 (ref)

$1.09 \quad(0.78-1.51) \quad 0.62$

1 (ref)

$0.78 \quad(0.52-1.19) \quad 0.25$

$0.63 \quad(0.40-0.98) \quad 0.04$

\begin{tabular}{|c|c|c|c|c|c|c|c|c|c|c|c|}
\hline 1 & (ref) & & 1 & (ref) & & 1 & (ref) & & 1 & (ref) & \\
\hline 1.18 & $(0.80-1.74)$ & 0.41 & 1.07 & $(0.70-1.64)$ & 0.76 & 0.97 & $(0.63-1.51)$ & 0.91 & 1.25 & $(0.85-1.85)$ & 0.26 \\
\hline 1 & (ref) & & 1 & (ref) & & 1 & (ref) & & 1 & (ref) & \\
\hline 1.09 & $(0.71-1.67)$ & 0.69 & 1.15 & $(0.70-1.88)$ & 0.58 & 1.06 & $(0.72-1.57)$ & 0.77 & 0.75 & $(0.50-1.11)$ & 0.15 \\
\hline 1.19 & $(0.66-2.16)$ & 0.56 & 1.14 & $(0.61-2.13)$ & 0.69 & 1.03 & $(0.58-1.82)$ & 0.93 & 0.57 & $(0.31-1.06)$ & 0.08 \\
\hline 1.13 & $(0.56-2.28)$ & 0.74 & 1.65 & $(0.77-3.55)$ & 0.20 & 1.43 & $(0.66-3.08)$ & 0.37 & 1.07 & $(0.52-2.20)$ & 0.86 \\
\hline
\end{tabular}

Children in household

$\begin{array}{llllllllllll}1 & \text { (ref) } & & 1 & \text { (ref) } & & 1 & \text { (ref) } & & 1 & \text { (ref) } & \\ 0.90 & (0.54-1.48) & 0.67 & 0.65 & (0.35-1.21) & 0.17 & 0.90 & (0.55-1.47) & 0.68 & 0.97 & (0.60-1.58) & 0.91\end{array}$


Household income

$£ 0-£ 10,400$
$£ 10,400-£ 20,800$
$£ 20,800-£ 31,200$
$£ 31,200-£ 52,000$
$£ 52,000+$

$\begin{array}{lll}1 & \text { (ref) } & \\ 1.48 & (0.76-2.92) & 0.25 \\ 1.48 & (0.74-2.98) & 0.27 \\ 1.30 & (0.65-2.60) & 0.46 \\ 1.38 & (0.65-2.93) & 0.40\end{array}$

1 (ref)

$1.51 \quad(0.74-3.11) \quad 0.26$

$1.76 \quad(0.87-3.58) \quad 0.12$

$2.01 \quad(0.98-4.11) \quad 0.06$

$1.48 \quad(0.63-3.47) \quad 0.36$
1 (ref)

$0.69 \quad(0.39-1.22) \quad 0.20$

$0.54 \quad(0.31-0.95) \quad 0.03$

$0.68 \quad(0.39-1.19) \quad 0.17$

$0.88 \quad(0.49-1.61) \quad 0.69$
1 (ref)

$0.49 \quad(0.29-0.83) \quad 0.01$

$0.57 \quad(0.34-0.96) \quad 0.04$

$0.86 \quad(0.26-0.94) \quad 0.57$

$0.49(0.79-1.56) \quad 0.03$

Education

$\begin{array}{lllllll}\text { Less than higher education } & 1 & \text { (ref) } & & 1 & \text { (ref) } & \\ \text { Higher education degree } & 1.29 & (0.92-1.83) & 0.142 & 0.95 & (0.64-1.42) & 0.81\end{array}$

1 (ref)

1 (ref)

$1.77 \quad(1.28-2.45) \quad<0.01$

$1.11(0.79-1.56) \quad 0.53$

Job impact

No

Yes

Caring responsibility

$\begin{array}{lllllll}\text { No } & 1 & \text { (ref) } & & 1 & \text { (ref) } & \\ \text { Yes } & 0.81 & (0.48-1.36) & 0.424 & 1.23 & (0.76-1.97) & 0.40\end{array}$

Affected usual healthcare

No

Yes

Impact on standard of living

$$
\begin{aligned}
& \text { Same or improved } \\
& \text { Worsened }
\end{aligned}
$$

$\begin{array}{lll}1 & \text { (ref) } & \\ 0.80 & (0.53-1.22) & 0.307\end{array}$

$1.23 \quad(0.76-1.97) \quad 0.40$

1 (ref)

$1.00 \quad(0.71-1.42) \quad 0.987$

1 (ref)

$1.07 \quad(0.73-1.57) \quad 0.73$

$0.99 \quad(0.66-1.47) \quad 0.949$
1 (ref)
1 (ref)

$1.13 \quad(0.74-1.73) \quad 0.56$
1 (ref)

$0.62(0.40-0.95) \quad 0.03$

1 (ref)

$0.71 \quad(0.45-1.13) \quad 0.15$

$1.33 \quad(0.88-1.99) \quad 0.17$
1 (ref)

$1.05 \quad(0.76-1.45) \quad 0.76$

$1.16 \quad(0.81-1.67) \quad 0.41$

1 (ref)

$0.84 \quad(0.60-1.17) \quad 0.29$

$0.95 \quad(0.66-1.37) \quad 0.78$

Note: bold indicates significance at the $95 \%$ level. 
Table 3. Preferences for lockdown features.

\begin{tabular}{|c|c|c|c|c|c|c|c|c|c|c|}
\hline & \multicolumn{5}{|c|}{ England } & \multicolumn{5}{|c|}{ Northern Ireland } \\
\hline & \multicolumn{3}{|c|}{ Parameter estimates } & \multicolumn{2}{|c|}{ MRS (excess deaths) } & \multicolumn{3}{|c|}{ Parameter estimates } & \multicolumn{2}{|c|}{ MRS (excess deaths) } \\
\hline & Coef. & $95 \% \mathrm{Cl}$ & $p$ value & MRS & $95 \% \mathrm{Cl}$ & Coef. & $95 \% \mathrm{Cl}$ & $p$ value & MRS & $95 \% \mathrm{Cl}$ \\
\hline Alternative Specific Constant & -0.15 & $(-0.11--0.20)$ & $<0.01$ & .. & .. & -0.16 & $(-0.21--0.10)$ & $<0.01$ & .. & .. \\
\hline Green restrictions & 0.01 & $(-0.04-0.06)$ & 0.75 & -0.49 & $(-0.91--0.07)$ & 0.00 & $(-0.05-0.06)$ & 0.87 & -0.04 & $(-0.48-0.40)$ \\
\hline Yellow restrictions & 0.19 & $(0.14-0.25)$ & $<0.01$ & -1.63 & $(-2.09--1.17)$ & 0.12 & $(0.05-0.18)$ & $<0.01$ & -0.91 & $(-1.38--0.43)$ \\
\hline Amber restrictions & 0.05 & $(-0.00-0.10)$ & 0.07 & -0.39 & $(-0.82-0.03)$ & 0.07 & $(0-01-0.12)$ & 0.02 & -0.51 & $(-0.94--0.07)$ \\
\hline Red restrictions & -0.25 & $(-0.20-0.30)$ & $<0.01$ & 2.09 & $(1.66-2.52)$ & -0.19 & $(-0.14--0.25)$ & $<0.01$ & 1.45 & $(1.00-1.89)$ \\
\hline Length (1 week increase) & -0.02 & $(-0.02--0.03)$ & $<0.01$ & 0.20 & $(0.15-0.26)$ & -0.03 & $(-0.03--0.02)$ & $<0.01$ & 0.19 & $(0.14-0.25)$ \\
\hline All healthcare postponed & -0.03 & $(-0.07-0.01)$ & 0.16 & 0.24 & $(-0.09-0.57)$ & -0.04 & $(-0.09-0.00)$ & 0.08 & 0.31 & $(-0.03-0.65)$ \\
\hline Some healthcare postponed & -0.03 & $(-0.07-0.01)$ & 0.16 & 0.24 & $(-0.09-0.57)$ & 0.00 & $(-0.05-0.04)$ & 0.85 & 0.03 & $(-0.31-0.38)$ \\
\hline None healthcare postponed & 0.06 & $(0.02-0.10)$ & 0.01 & -0.48 & $(-0.88--0.14)$ & 0.05 & $(-0.00-0.09)$ & 0.06 & -0.34 & $(-0.69-0.01)$ \\
\hline Excess deaths ( 1 out of 10,000 increase) & -0.12 & $(-0.12--0.11)$ & $<0.01$ & - & - & -0.13 & $(-0.14--0.14)$ & $<0.01$ & - & - \\
\hline Ability to spend ( $10 \%$ decrease) & 0.10 & $(0.08-0.13)$ & $<0.01$ & -0.88 & $(-1.11--0.66)$ & 0.12 & $(0.09-0.15)$ & 0.00 & 0.19 & $(-1.12--0.64)$ \\
\hline Job loss (1 out of 100 increse) & -0.02 & $(-0.03--0.02)$ & $<0.01$ & 0.20 & $(0.17-0.23)$ & -0.03 & $(-0.03--0.02)$ & $<0.01$ & 1.20 & $(0.16-0.22)$ \\
\hline SD Alternative Specific Constant & 0.00 & $(-0.10-0.10)$ & 1.00 & .. & .. & 0.00 & $(-0.13-0.13)$ & 0.99 & .. & .. \\
\hline Log likelihood & \multicolumn{5}{|c|}{-5167.934} & \multicolumn{5}{|c|}{-3811.386} \\
\hline Observations & \multicolumn{5}{|l|}{8896} & \multicolumn{5}{|l|}{6784} \\
\hline
\end{tabular}


Table 3 continued. Preferences for lockdown features.

\begin{tabular}{|c|c|c|c|c|c|c|c|c|c|c|}
\hline & \multicolumn{5}{|c|}{ Scotland } & \multicolumn{5}{|c|}{ Wales } \\
\hline & \multicolumn{3}{|c|}{ Parameter estimates } & \multicolumn{2}{|c|}{ MRS (excess deaths) } & \multicolumn{3}{|c|}{ Parameter estimates } & \multicolumn{2}{|c|}{ MRS (excess deaths) } \\
\hline & Coef. & $95 \% \mathrm{Cl}$ & $\mathrm{p}$ value & MRS & $95 \% \mathrm{Cl}$ & Coef. & $95 \% \mathrm{Cl}$ & $\mathrm{p}$ value & MRS & $95 \% \mathrm{Cl}$ \\
\hline Alternative Specific Constant & -0.17 & $(-0.22--0.12)$ & $<0.01$ & .. & .. & -0.21 & $(-0.26-0.16)$ & $<0.01$ & .. & .. \\
\hline Green restrictions & 0.14 & $(0.08-0.19)$ & $<0.01$ & -1.15 & $(-1.64--0.67)$ & 0.06 & $(0.01-0.11)$ & 0.03 & -0.49 & $(-0.89--0.10)$ \\
\hline Yellow restrictions & 0.13 & $(0.08-0.19)$ & $<0.01$ & -0.92 & $(-1.31--0.53)$ & 0.06 & $(0.01-0.11)$ & 0.02 & -0.48 & $(-0.89--0.06)$ \\
\hline Amber restrictions & 0.03 & $(-0.02--0.08)$ & 0.25 & -0.20 & $(-0.05-0.142)$ & 0.04 & $(-0.01-0.09)$ & 0.08 & -0.33 & $(-0.71-0.05)$ \\
\hline Red restrictions & -0.30 & $(-0.35--0.25)$ & $<0.01$ & 2.04 & $(1.68-2.41)$ & -0.17 & $(-0.2--0.11)$ & $<0.01$ & 1.25 & $(0.86-1.65)$ \\
\hline Length (weeks) & -0.03 & $(-0.04--0.03)$ & $<0.01$ & 0.22 & $(0.18-0.27)$ & -0.03 & $(-0.03--0.02)$ & $<0.01$ & 0.21 & $(0.17-0.26)$ \\
\hline All healthcare postponed & -0.01 & $(-0.05-0.03)$ & 0.53 & 0.09 & $(-0.19-0.37)$ & -0.03 & $(-0.07-0.01)$ & 0.17 & 0.21 & $(-0.09-0.51)$ \\
\hline Some healthcare postponed & -0.02 & $(-0.06-0.02)$ & 0.27 & 0.16 & $(-0.12-0.44)$ & 0.01 & $(-0.03-0.05)$ & 0.65 & -0.07 & $(-0.38-0.24)$ \\
\hline None healthcare postponed & 0.04 & $(-0.00-0.08)$ & 0.09 & 0.18 & $(-0.10-0.47)$ & 0.02 & $(-0.02-0.06)$ & 0.38 & -0.14 & $(-0.46-0.18)$ \\
\hline Excess deaths (increase 1 out of 10,000 ) & -0.15 & $(-0.15--0.14)$ & $<0.01$ & - & - & -0.13 & $(-0.13--0.12)$ & $<0.01$ & - & - \\
\hline Ability to spend ( $10 \%$ decrease) & 0.09 & $(0.07-0.12)$ & $<0.01$ & -0.62 & $(-0.82--0.42)$ & 0.10 & $(0.07-0.13)$ & $<0.01$ & -0.76 & $(-0.98--0.54)$ \\
\hline Job loss (1 out of 100$)$ & -0.03 & $(-0.04--0.03)$ & $<0.01$ & 0.22 & $(0.20-0.24)$ & -0.03 & $(-0.03--0.02)$ & $<0.01$ & 0.20 & $(0.18-0.23)$ \\
\hline SD Alternative Specific Constant & 0.00 & $(-0.10-0.10)$ & 0.99 & . & .. & 0.00 & $(-0.10-0.10)$ & 0.99 &.. & .. \\
\hline Log likelihood & -4890 & .047 & & & & -4925 . & 654 & & & \\
\hline Observations & 9144 & & & & & 8784 & & & & \\
\hline
\end{tabular}

Note: Coef. $=$ mean parameter coefficient estimate. $\mathrm{Cl}=$ Confidence Interval. $\mathrm{SD}=$ Standard Deviation. MRS $=$ Marginal rates of substitution Categorical variables were effects coded to allow for a preference parameter to be estimated for all levels of the feature[29]. 


\section{Discussion}

The elicitation of public values and trade-offs for different lockdown features can help guide government policies during a pandemic. We found evidence that four out of five respondents were willing to accept an increase in excess deaths for relaxations in lockdown restrictions. This suggests that as the governments of the devolved nations consider easing lockdown, the public will be willing to accept an increase in excess deaths. We also estimated acceptable excess deaths for such relaxations.

With the roll-out of pharmaceutical interventions and the increase in data available to model the impact of changes in restrictions, our model can help inform policy makers about what lockdown policies are acceptable given the estimated trade-offs. We found that respondents in England are the most averse to the introduction of short circuitbreaker-type lockdowns, thus accepting a higher number of excess deaths to avoid them. In contrast, these strict lockdowns were more palatable to respondents in Wales.

Trade-off values can also be interpreted as the number of lives that need to be saved if a less preferred and expectedly stricter lockdown is implemented. Our model can be used to assess whether the expected health benefits in terms of a reduction in the number of excess deaths outweigh costs in terms of increased restrictions. As an example, modelling by Ferguson et al. (2020) contended that a one-week earlier strict lockdown in England during COVID-19's first wave would have saved 20,000 lives.[28] Our findings suggest that the number of acceptable deaths in England for a one-week strict (red level restrictions) lockdown is 2.53 out of 10,000 , or 14,170 lives, which is less than the number of lives that would have been saved (see online Supplemental Material p.14 for details). Thus, based on these results, the benefits of introducing an earlier lockdown would have outweighed the costs in terms of lockdown restrictions. These insights can be useful as UK governments consider easing lockdown restrictions or the introduction of new ones if future infection waves occur.

Whilst we limited our analysis to consider acceptable excess deaths, a strength of our model is that it can be used to determine value in terms of other features included, i.e. acceptable reductions in spending or job losses associated with a particular lockdown scenario. We found that respondents in Scotland were less sensitive to losses in their own spending ability compared to other nations. For example, the average acceptable loss in spending ability for a four-week red level lockdown in Scotland is $49 \%$, while in England it is $36 \%$, Northern Ireland it is $29 \%$, and Wales $30 \%$. A detailed calculation of these MRS can be found in the online Supplemental Material (p. 16). Thus, a targeted compensation instrument could target other economic consequences, such as joblessness, in Scotland and consumer spending ability in the other nations.

Our study is not exempt from limitations. A potential limitation is that individual's preferences regarding the features of lockdown may be evolving. Until March 2020, respondents would not have experienced a lockdown. However, we conducted our survey in October-December 2020, hence all respondents would have experience of the first lockdown. The study was, however, conducted before the second lockdown. 
medRxiv preprint doi: https://doi.org/10.1101/2021.10.12.21264883; this version posted October 14, 2021. The copyright holder for this preprint (which was not certified by peer review) is the author/funder, who has granted medRxiv a license to display the preprint in perpetuity.

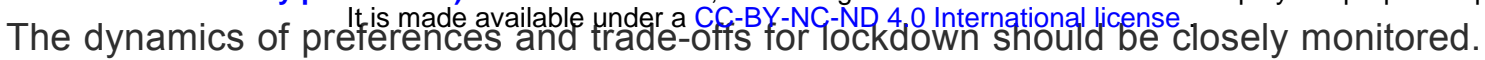

Another possible limitation is that we identified respondents as excess death minimisers if they chose the option with the minimum number of deaths in all eight choice tasks. This response pattern could also represent a decision-heuristic for respondents to complete the tasks. It is also possible that respondents are considering excess deaths, and trading, but the combination of feature levels results in the option with the lowest number of excess deaths. Either way, this suggests our estimate of $80 \%$ of respondents being willing to trade would be an underestimate. Further, we estimated trade-offs across the entire sample, allowing for the possibility that such responders were traders. We have not attempted to explain preference heterogeneity across nor within nations. Our study did not look at the relative importance of the different dimensions of lockdown restrictions (shelter, socialising, non-essential trips, schools and youth activities, non-essential businesses and outdoor activities). Future work could use a DCE to explore this; given current discussions around international travel, this dimension could be included. We focused on the preferences of the public; future research could explore the preferences of policy makers and health professionals.

\section{Conclusions}

In this study we have provided new insight into preferences for lockdown policies across the four UK nations using a DCE. The majority of respondents from all four devolved nations were willing to accept an increase in excess deaths for relaxation in lockdown restrictions. Respondents from England were more willing to accept an increase in excess deaths, followed by Scotland, Northern Ireland and Wales. Our model can also be used to estimate the reduction in excess deaths required to justify increasing lockdown restrictions. Whilst we focused on excess deaths, trade-offs could also be estimated in terms of acceptable changes in spending power and job losses, as well as combinations of these features. Such analysis will help identify which levers best support lockdown strategies whilst maintaining public confidence and maximising compliance. 
medRxiv preprint doi: https://doi.org/10.1101/2021.10.12.21264883; this version posted October 14, 2021. The copyright holder for this preprint (which was not certified by peer review) is the author/funder, who has granted medRxiv a license to display the preprint in perpetuity. Acknowledgements It is made available under a CC-BY-NC-ND 4.0 International license.

We want to thank the members of the public who took part in the think aloud interviews as part of the study development stage. We thank the members of the Stakeholder Advisory Group for their continued involvement in the research study. We also thank $\mathrm{Dr}$ Dwayne Boyers for his internal review of the study protocol and Professor Vikki Entwistle for comments on the protocol.

\section{Contributors}

MR, VW, MG, RAS \& LEL-R conceptualised the study, contributed to the overall design of the survey experiment and contributed to the interpretation of the data. MG and RAS carried out the think-aloud interviews as part of the developmental work. LEL-R undertook the analysis, including the $R$ programming of the statistical models and is the study's guarantor. MR reviewed the statistical model and contributed to the analysis of the data. SP and DP contributed comments to the development of the protocol, and discussion of public health implications and helped shape the overall interpretation. All authors approved the final protocol. All authors had access to all the data, contributed to the writing of the paper and had final responsibility for the decision to submit for publication. The corresponding author attests that all listed authors meet authorship criteria and that no others meeting the criteria have been omitted.

\section{Funding}

This study was funded by the Health Economics Research Unit, the University of Aberdeen, and the Chief Scientist Office of the Scottish Government Health and Social Care Directorates. Award/Grant number is not applicable. The funders had no role in considering the study design or in the collection, analysis, or interpretation of data, the writing of the report, or the decision to submit the article for publication.

\section{Declaration of interests}

All authors have completed the ICMJE uniform disclosure form at www.icmje.org/coi_disclosure.pdf (available on request from the corresponding author) and declare no support from any organisation for the submitted work; no financial relationships with any organisations that might have an interest in the submitted work in the previous three years; no other relationships or activities that could appear to have influenced the submitted work.

\section{Ethical approval}

The study received ethical approval from the University of Aberdeen's College of Life Sciences and Medicine Ethical Review Board (Reference CERB/2020/6/1974). All participants provided informed consent.

\section{Data sharing}

Anonymised cross-sectional data from the analysis can be made available by the corresponding author after the authors' review of reasonable requests. The published protocol can be found at: https://bmjopen.bmj.com/content/10/11.

\section{Dissemination to participants and related patient and public communities}

The results have been and will be presented at national and international conferences. Dissemination plans to inform the community of this study's results include social 
medRxiv preprint doi: https://doi.org/10.1101/2021.10.12.21264883; this version posted October 14, 2021. The copyright holder for this preprint (which was not certified by peer review) is the author/funder, who has granted medRxiv a license to display the preprint in perpetuity.

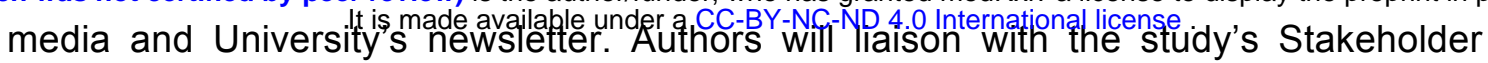
Advisory Group to ensure maximum policy impact of the study's findings.

\section{Transparency}

The corresponding author affirms that this manuscript is an honest, accurate, and transparent account of the study being reported; that no important aspects of the study have been omitted; and that any discrepancies from the study as planned (and, if relevant, registered) have been explained. 
(1) Ferguson NM, Laydon D, Nedjati-Gilani G, et al. Impact of non-pharmaceutical interventions (NPIs) to reduce COVID-19 mortality and healthcare demand. Imperial College COVID-19 Response Team. 2020;20.

(2) Hale T, Angrist N, Goldszmidt R, et al. A global panel database of pandemic policies (Oxford covid-19 government response tracker). Nature Human Behaviour 2021:1-10.

(3) Mahtani KR, Heneghan C, Aronson JK. What is the evidence for social distancing during global pandemics? A rapid summary of current knowledge. The Centre for Evidence-Based Medicine, available from https://www.cebm.net/covid-19/what-is-theevidence-for-social-distancing-during-global-pandemics/accessed on 2020;5.

(4) Centre for Disease Control and Prevention. Social Distancing. November 2020. Available at: https://www.cdc.gov/coronavirus/2019-ncov/prevent-getting-sick/socialdistancing.html Accessed April 2020.

(5) Mahase E. Covid-19: UK starts social distancing after new model points to 260000 potential deaths. BMJ 2020;368:m1089

(6) Scientific Advisory Group for Emergencies. Scientific evidence supporting the government response to coronavirus (COVID-19). 2021. Available at:

https://www.gov.uk/government/collections/scientific-evidence-supporting-thegovernment-response-to-coronavirus-covid-19\#spi-b-background-papers. Accessed March, 2021.

(7) Colbourn T. COVID-19: extending or relaxing distancing control measures. Lancet Public Health 2020;5(5):e236-7.

(8) Venkatesh A, Edirappuli S. Social distancing in covid-19: what are the mental health implications? BMJ 2020;6;369:m1379.

(9) Koren M, Pető R. Business disruptions from social distancing. PLoS ONE 2020;15(9): e0239113.

(10) Coibion O, Gorodnichenko Y, Weber M. The cost of the covid-19 crisis: Lockdowns, macroeconomic expectations, and consumer spending. National Bureau of Economic Research 2020:w27141

(11) Chetty R, Friedman J, Hendren N, et al. How did covid-19 and stabilization policies affect spending and employment? a new real-time economic tracker based on private sector data. NBER working paper 2020:w27431.

(12) World Health Organization. Coronavirus disease (COVID-19) pandemic. 2021; Available at: https://www.who.int/emergencies/diseases/novel-coronavirus-2019. Accessed March, 2020.

(13) Cabinet Office. Overarching Government Strategy to Respond to Pandemic Influenza - Analysis of Scientific Evidence Base. Available at: https://assets.publishing. service.gov.uk/government/uploads/system/uploads/attachme nt_data/file/61968/flu_pandemic_science_paper1.pdf. Accessed April, 2021. 
medRxiv preprint doi: https://doi.org/10.1101/2021.10.12.21264883; this version posted October 14, 2021. The copyright holder for this preprint (which was not certified by peer review) is the author/funder, who has granted medRxiv a license to display the preprint in perpetuity.

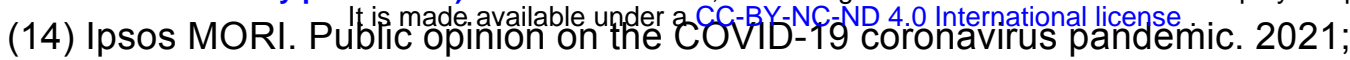

Available at: https://www.ipsos.com/ipsos-mori/en-uk/public-opinion-covid-19coronavirus-pandemic. Accessed February, 2021.

(15) University College London. Understanding the psychological and social impact of the pandemic. 2021; Available at: https://www.covidsocialstudy.org/. Accessed February, 2021.

(16) Williams SN, Armitage CJ, Tampe T, et al. Public perceptions and experiences of social distancing and social isolation during the COVID-19 pandemic: A UK-based focus group study. BMJ Open 2020;10(7):e039334.

(17) Bank of England. The UK economy: Insights from the Bank of England's Citizens' Panels. 2020; Available at: https://www.bankofengland.co.uk/get-involved/citizenspanels/the-uk-economy-insights-from-the-bank-of-englands-citizens-panels. Accessed February, 2021.

(18) The Scottish Parliament. Covid-19: Citizens' Panel. 2021 Available at: https://www.parliament.scot/parliamentarybusiness/CurrentCommittees/116947.aspx. Accessed March, 2021.

(19) Meyerowitz-Katz G, Merone L. A systematic review and meta-analysis of published research data on COVID-19 infection-fatality rates. International Journal of Infectious Diseases 2020;101:138-148

(20) Cook AR, Zhao X, Chen MIC, et al. Public preferences for interventions to prevent emerging infectious disease threats: a discrete choice experiment. BMJ Open 2018;8:e017355. doi: 10.1136/bmjopen-2017-017355

(21) Johnson J, Howard K, Wilson A, et al. Public preferences for One Health approaches to emerging infectious diseases: a discrete choice experiment. Social Science \& Medicine 2019;228:164-171.

(22) Genie MG, Loría-Rebolledo LE, Paranjothy S, et al. Understanding public preferences and trade-offs for government responses during a pandemic: a protocol for a discrete choice experiment in the UK. BMJ Open 2020;10(11):e043477.

(23) Bech M, Kjaer T, Lauridsen J. Does the number of choice sets matter? Results from a web survey applying a discrete choice experiment. Health Economics $2011 ; 20(3): 273-286$.

(24) Rose JM, Bliemer MC. Sample size requirements for stated choice experiments. Transportation 2013;40(5):1021-41.

(25) Ryan M, Krucien N, Hermens F. The eyes have it: Using eye tracking to inform information processing strategies in multi-attributes choices. Health Economics 2018;27(4):709-721.

(26) Lancsar E, Fiebig DG, Hole AR. Discrete choice experiments: a guide to model specification, estimation and software. Pharmacoeconomics 2017;35(7):697-716.

(27) Kolenikov S. Calibrating survey data using iterative proportional fitting (raking). The Stata Journal 2014;14(1):22-59. 
medRxiv preprint doi: https://doi.org/10.1101/2021.10.12.21264883; this version posted October 14, 2021. The copyright holder for this preprint (which was not certified by peer review) is the author/funder, who has granted medRxiv a license to display the preprint in perpetuity.

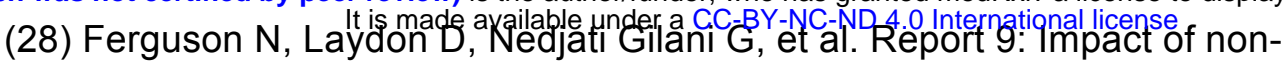
pharmaceutical interventions (NPIs) to reduce COVID19 mortality and healthcare demand. 2020.

(29) Bech M, Gyrd-Hansen D. Effects coding in discrete choice experiments. Health Economics 2005;14(10):1079-1083. 
England

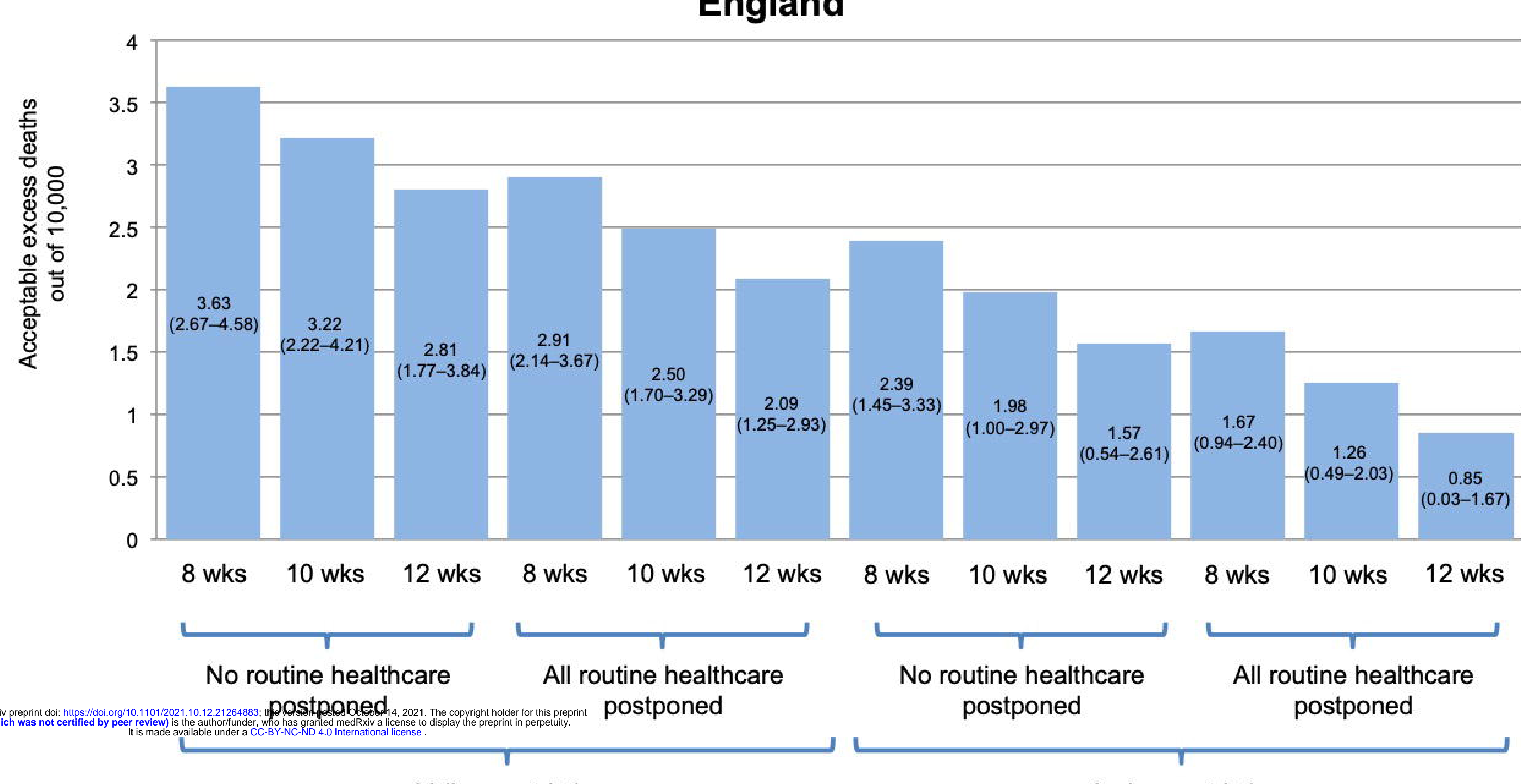

Yellow restrictions

Scotland

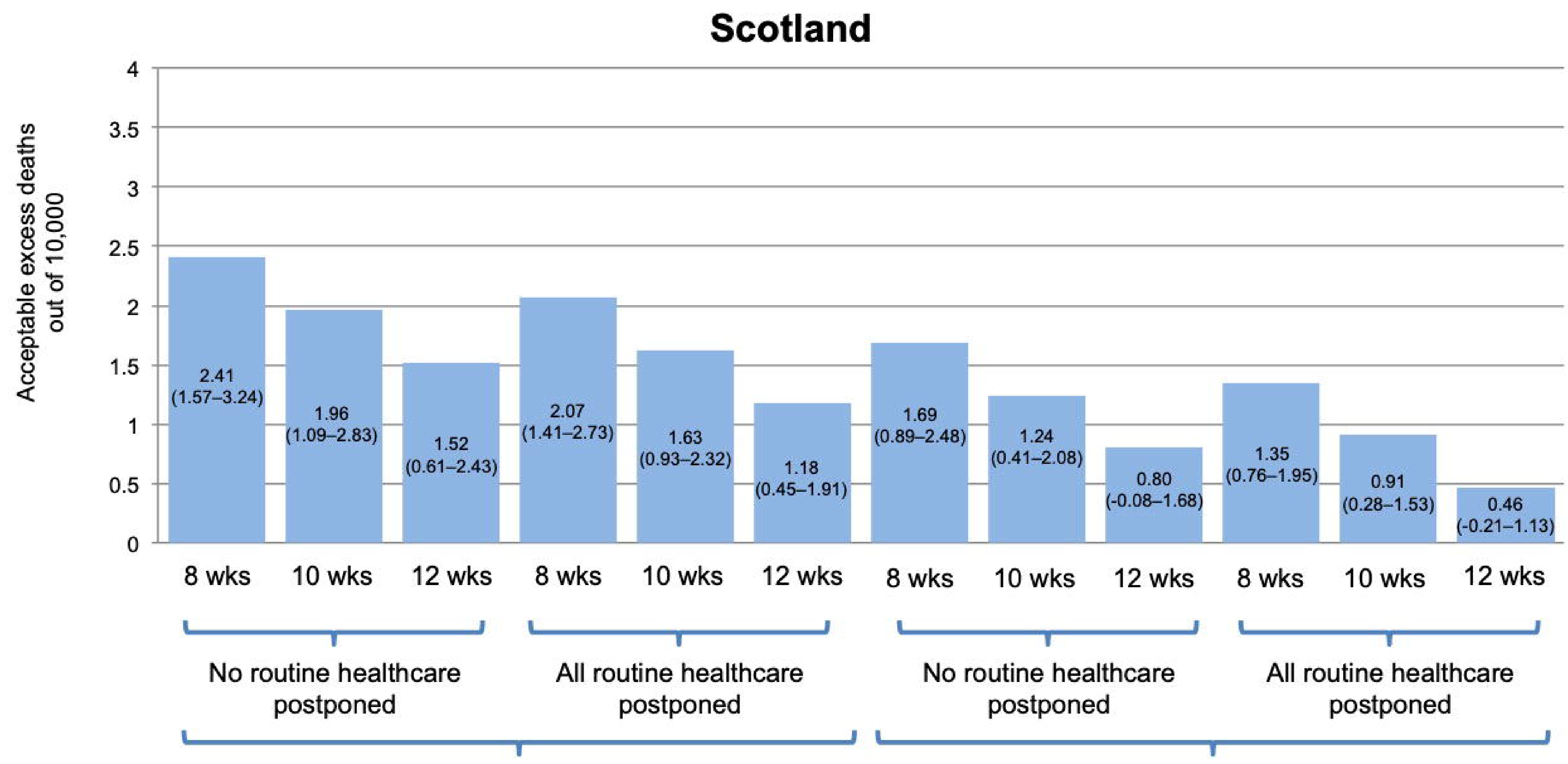

Yellow restrictions

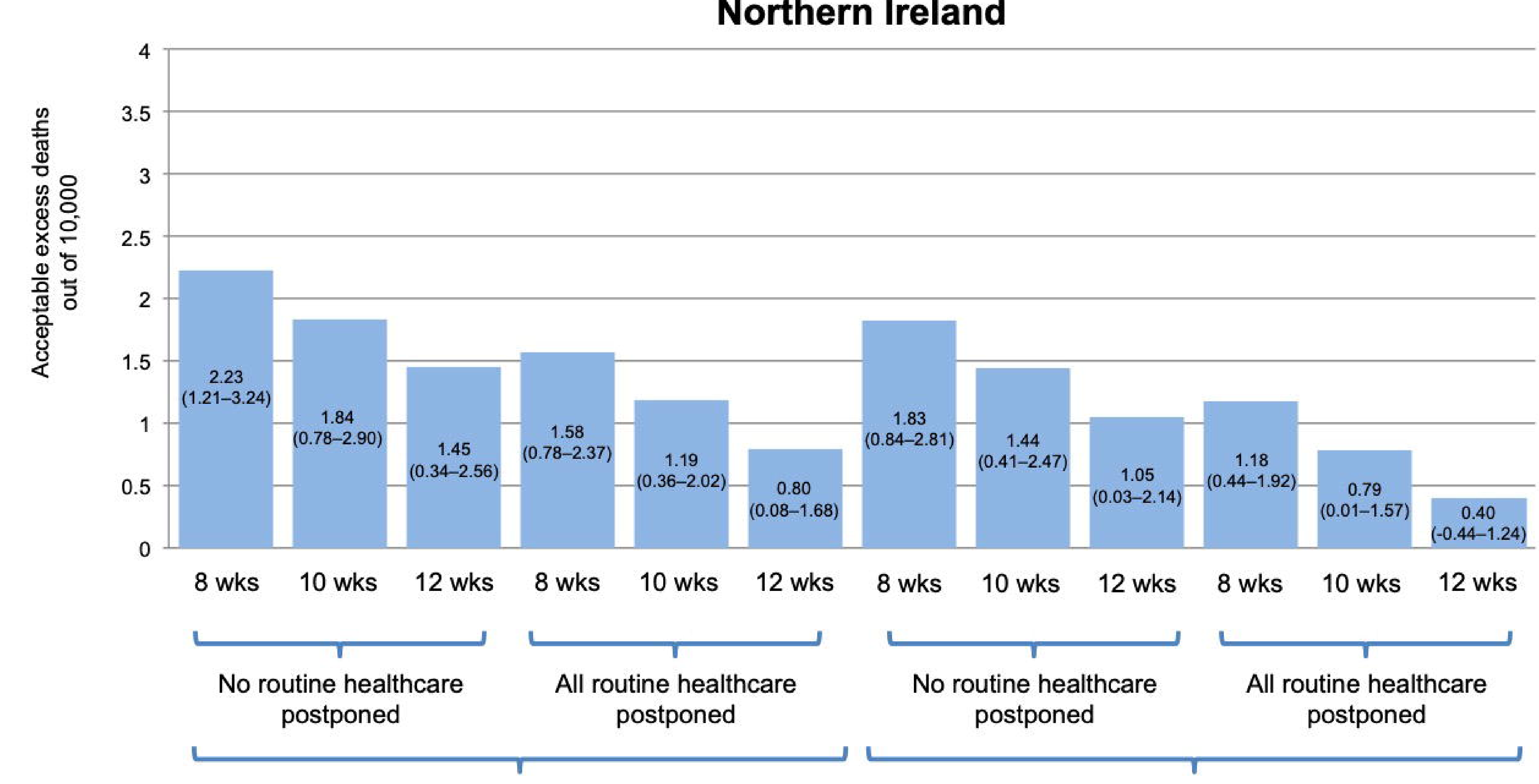

Yellow restrictions

Amber restrictions

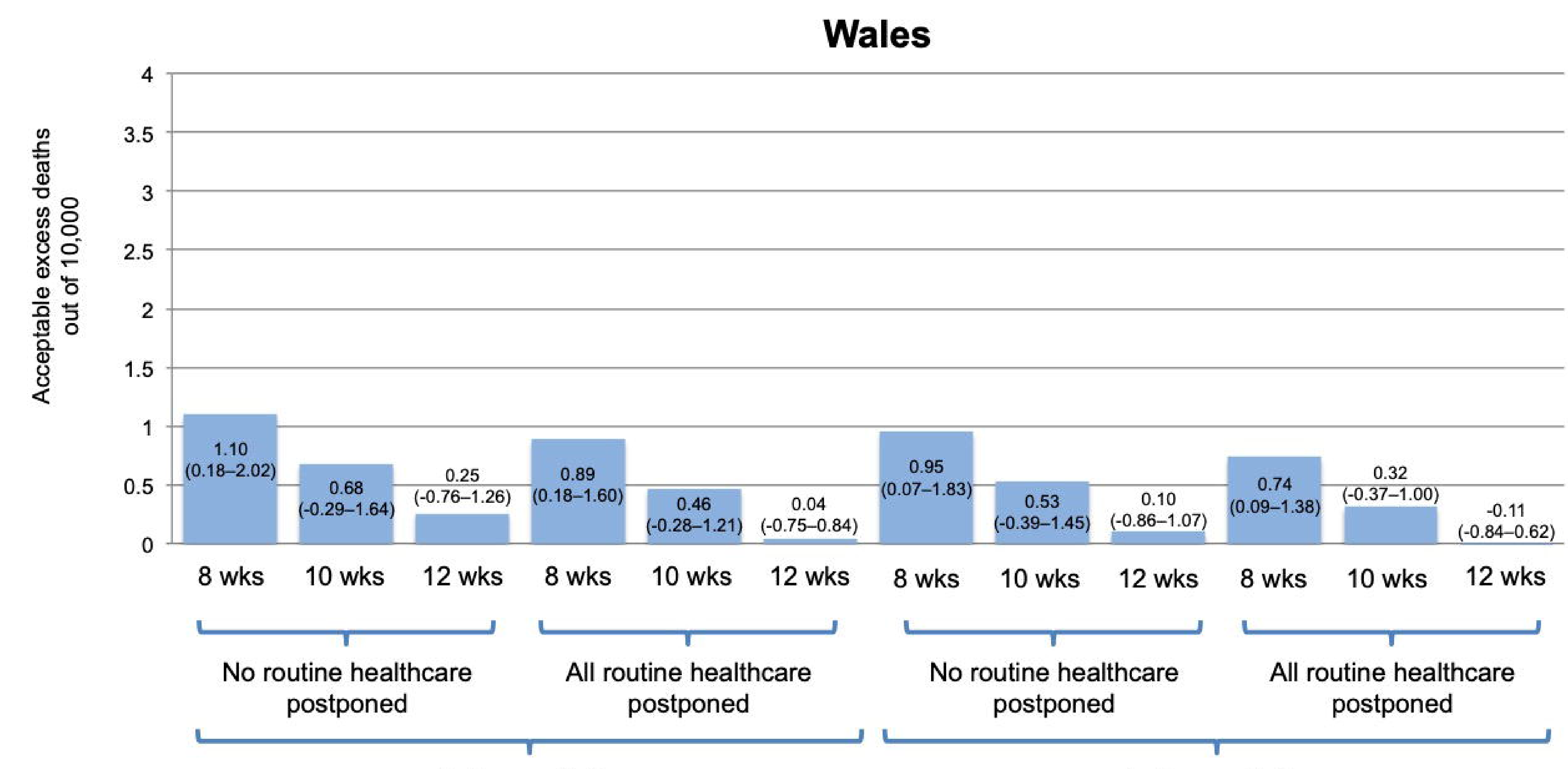

Yellow restrictions 


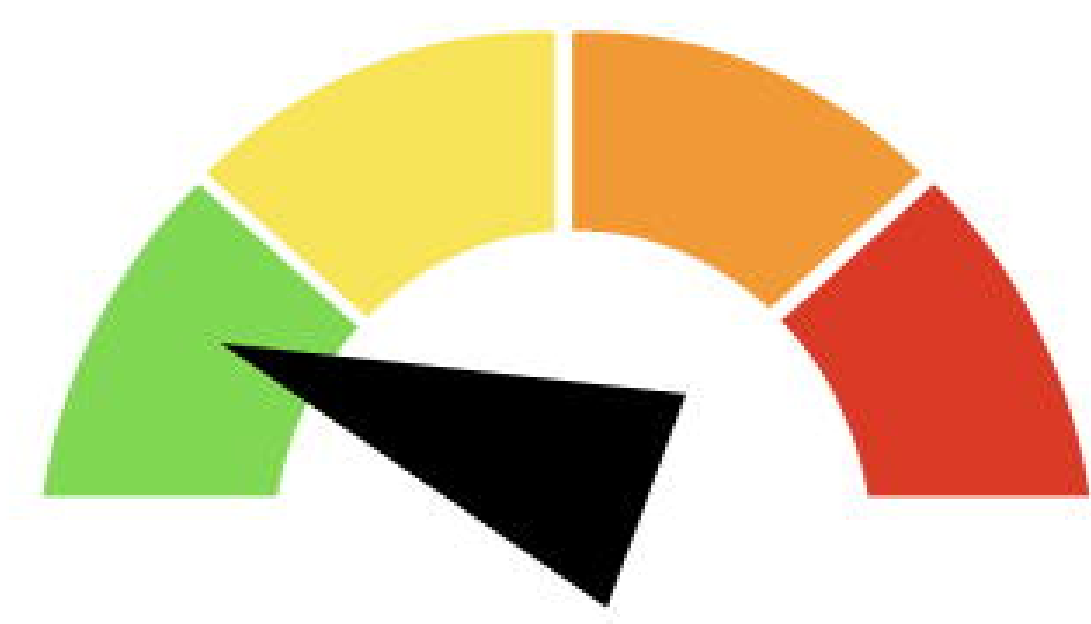

\section{Green Restrictions}

Shelter: everyone can interact with others.

Socialising: gatherings of up to 100 people.

Non-essential trips: are allowed.

Schools and youth activities: are open.

Non-essential businesses: operate under limited social distancing.

Outdoor leisure activities: are allowed.

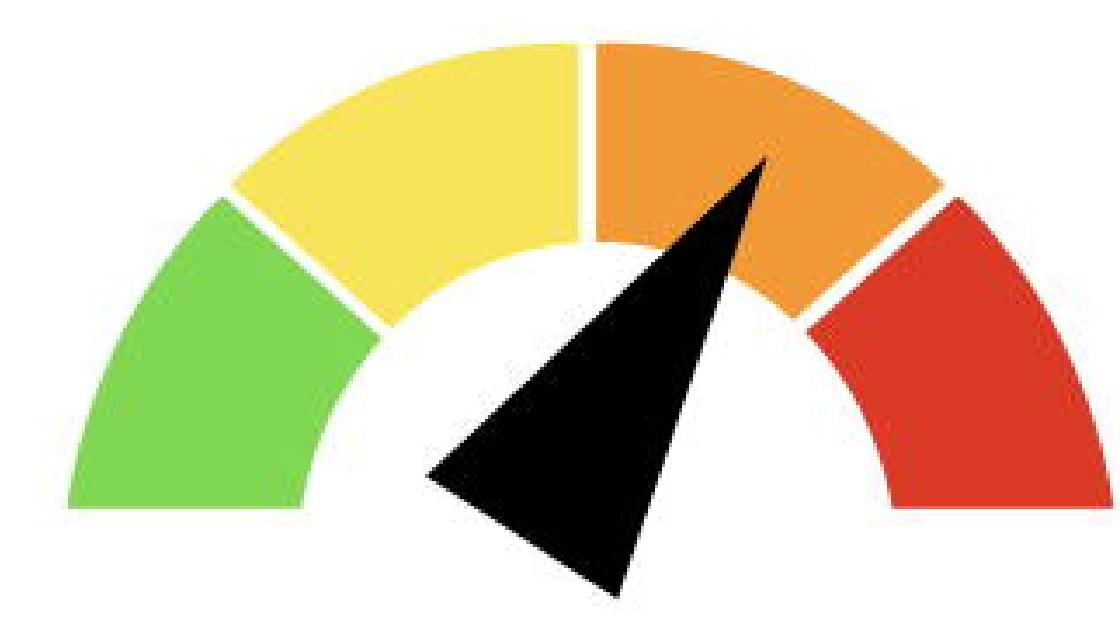

Amber Restrictions

Shelter: vulnerable people stay at home with no visitors.

Socialising: gatherings of up to 10 people.

Non-essential trips: should be minimised.

Schools and youth activities: are closed.

Non-essential businesses: operate under strict

distancing with limited capacity.

Outdoor leisure activities: are allowed.

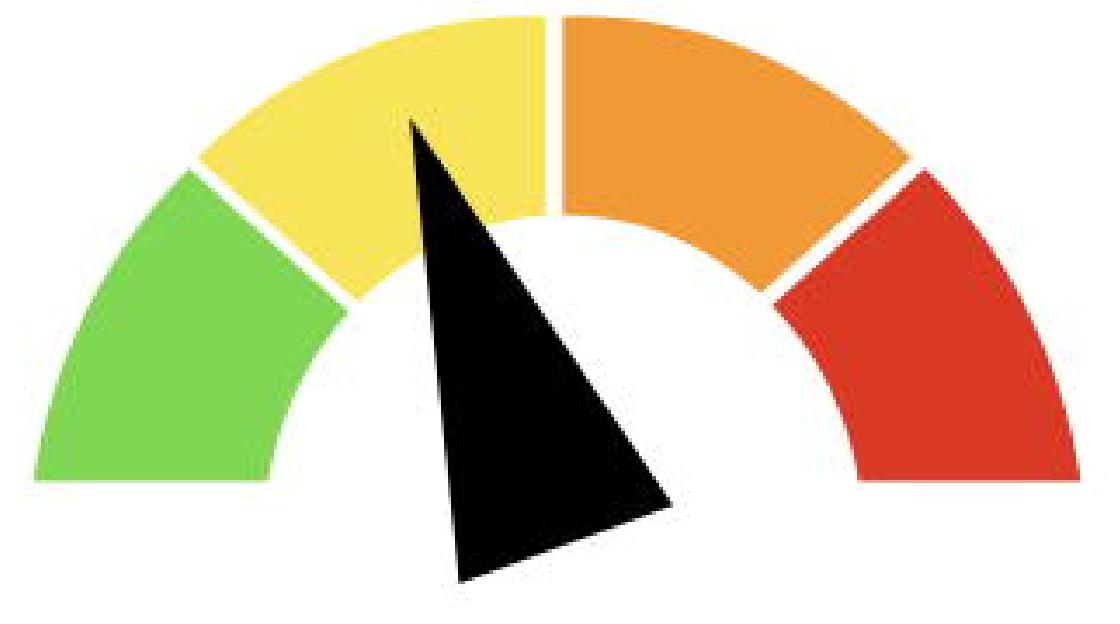

Yellow Restrictions

Shelter: vulnerable people should stay at home.

Socialising: gatherings of up to 50 people.

Non-essential trips: are allowed.

Schools and youth activities: are open.

Non-essential businesses: operate under moderate social distancing.

Outdoor leisure activities: are allowed.

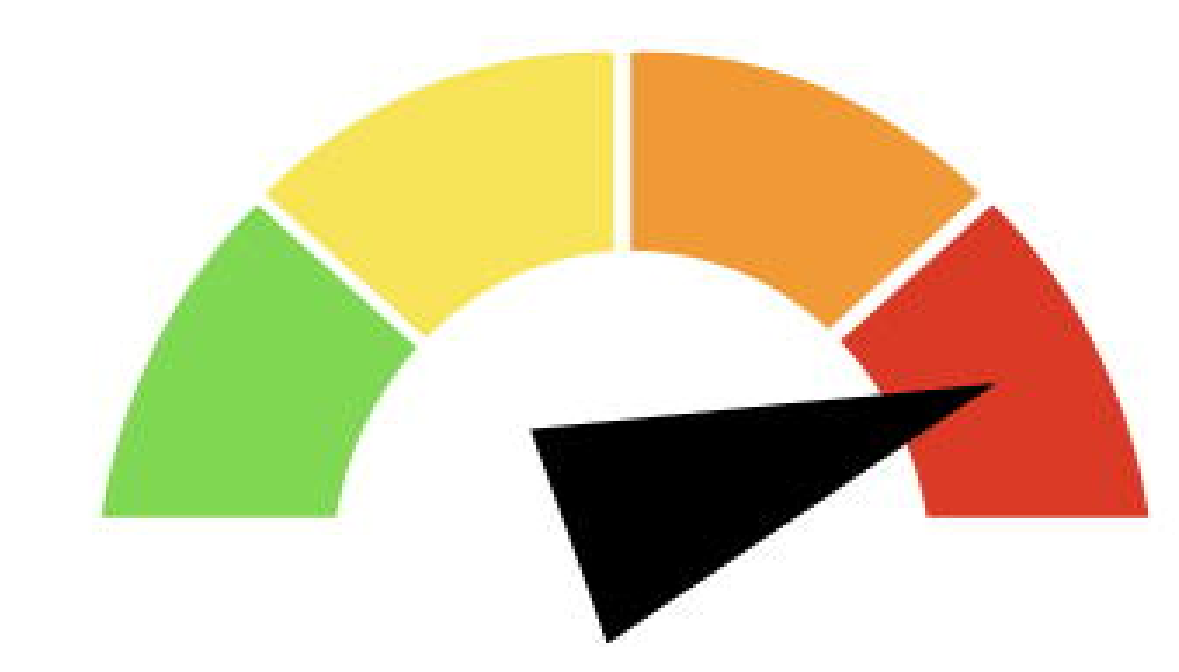

\section{Red Restrictions}

Shelter: everyone should stay at home with no visitors. Socialising: no gatherings beyond your own household. Non-essential trips: not allowed.

Schools and youth activities: are closed.

Non-essential businesses: remain closed.

Outdoor leisure activities: are not allowed. 


\section{Scenario 1}

This is the type of lockdown:

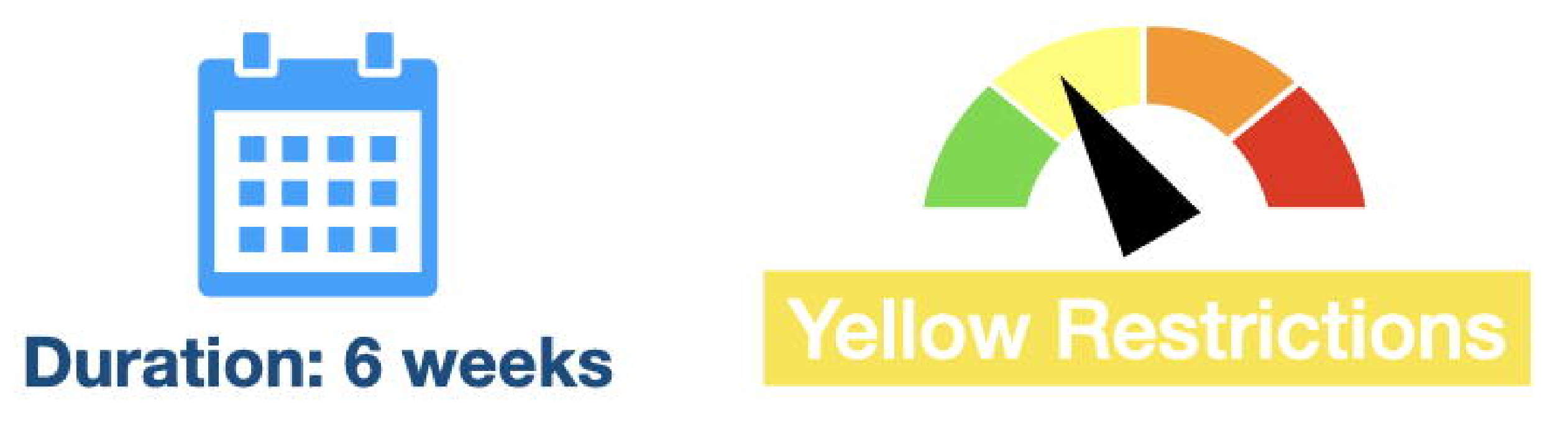

\section{Some planned procedures} postponed
Likely health impacts:

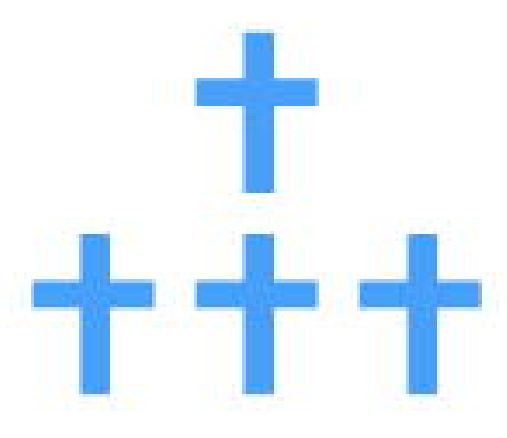

600 in 10,000 infected

4 in 10,000 additional people die

Likely economic impacts:
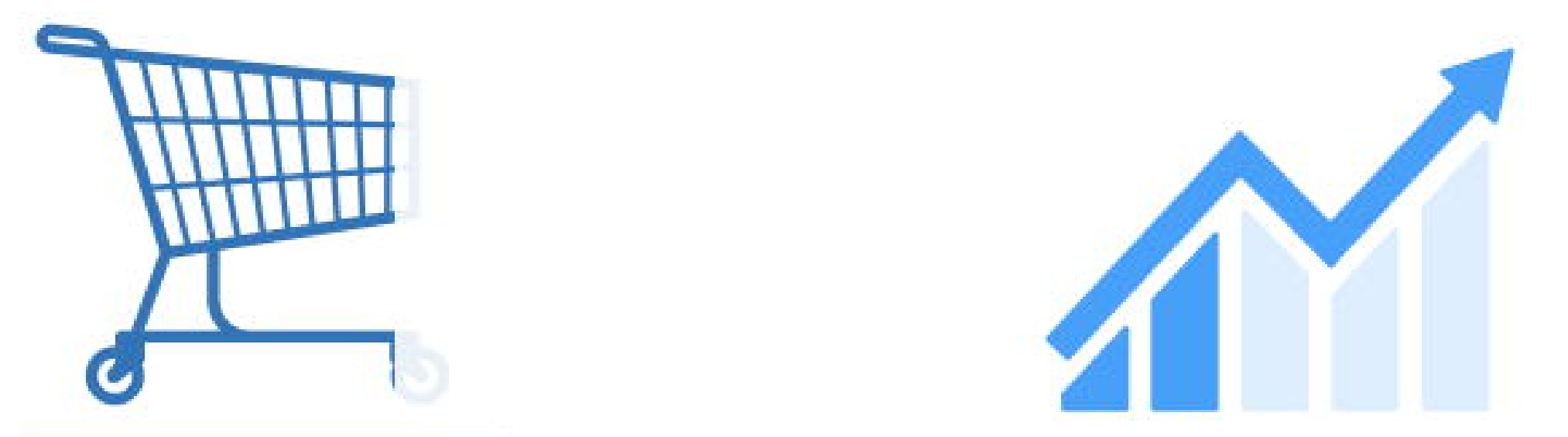

You can buy $90 \%$ of trolley

\section{Scenario 2}

This is the type of lockdown:

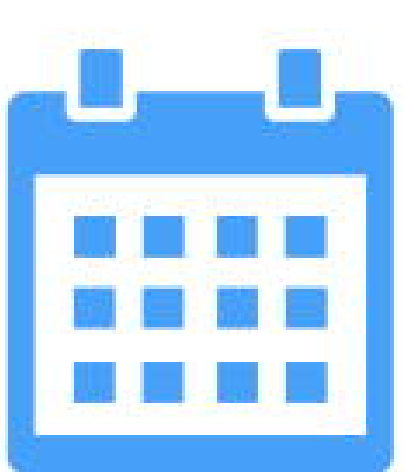

Duration: 10 weeks

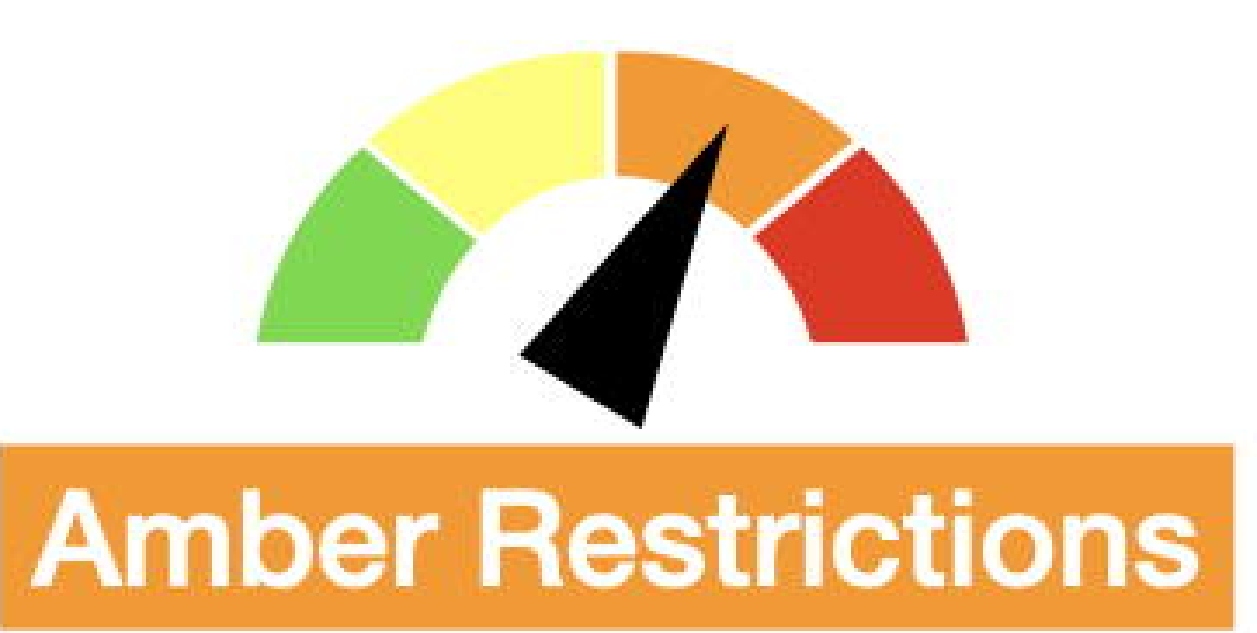

\section{All planned procedures} postponed

Likely health impacts:

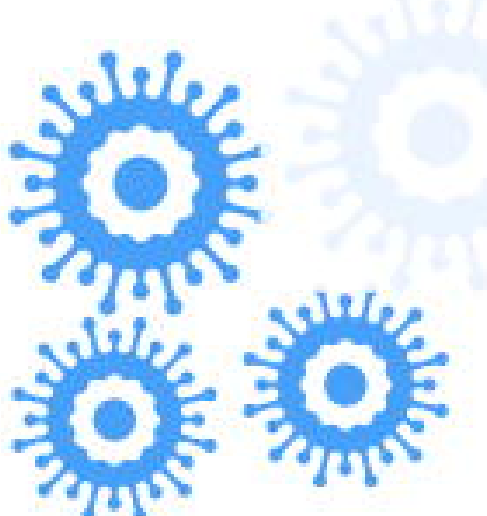

1,300 in 10,000 infected

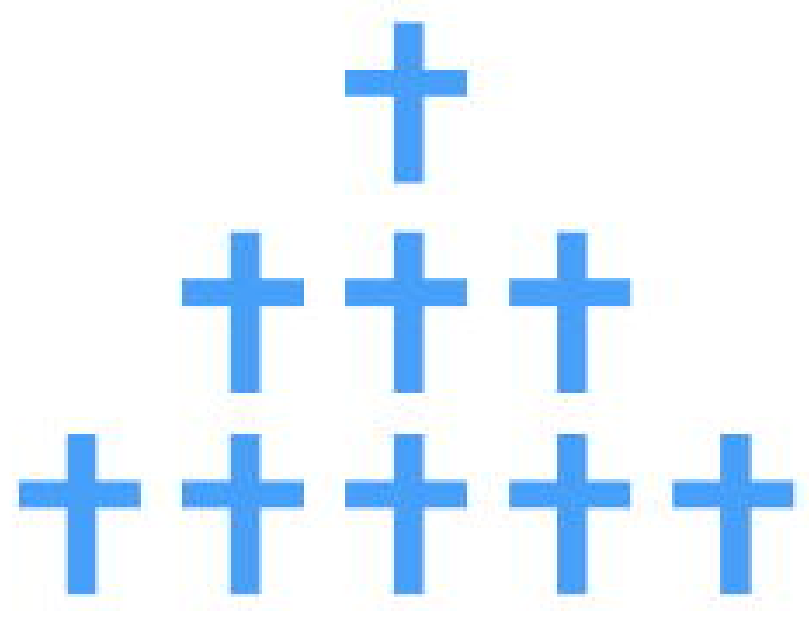

9 in 10,000 additional people die

Likely economic impacts:
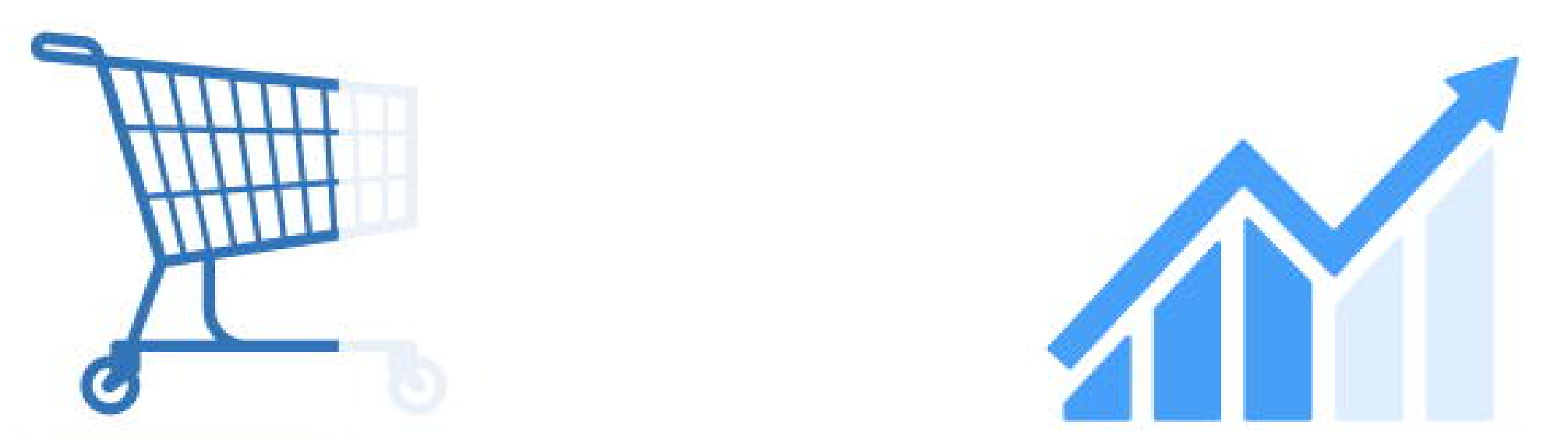

You can buy $80 \%$ of trolley 